\title{
Carbon Monoxide Poisoning and Chronic Kidney Disease Risk: A Nationwide, Population-Based Study
}

\author{
Kuang-Yu Wei ${ }^{a, b}$ Chen-Yi Liao ${ }^{a, c}$ Chi-Hsiang Chung d,e Fu-Huang Lin ${ }^{d}$ \\ Chang-Huei Tsaof,g,h Chien-An Sun ${ }^{\text {h, i }}$ Kuo-Cheng Lu ${ }^{\mathrm{i}, j}$ Wu-Chien Chien ${ }^{\mathrm{e}, \mathrm{f}, \mathrm{g}}$ \\ Chia-Chao $\mathrm{Wu}^{\mathrm{a}, \mathrm{k}}$ \\ aDivision of Nephrology, Department of Internal Medicine, Tri-Service General Hospital, National Defense Medical \\ Center, Taipei, Taiwan; 'bepartment of Internal Medicine, Division of Nephrology and Transplantation, Erasmus MC, \\ University Medical Center Rotterdam, Rotterdam, The Netherlands; 'Division of Nephrology, Department of Internal \\ Medicine, Kaohsiung Armed Forces General Hospital, Kaohsiung, Taiwan; ${ }^{d}$ School of Public Health, National Defense \\ Medical Center, Taipei, Taiwan; 'Taiwanese Injury Prevention and Safety Promotion Association, Taipei, Taiwan; \\ fDepartment of Medical Research, Tri-Service General Hospital, National Defense Medical Center, Taipei, Taiwan; \\ ${ }^{9}$ Graduate Institute of Life Sciences, National Defense Medical Center, Taipei, Taiwan; 'Department of Public Health, \\ College of Medicine, Fu-Jen Catholic University, New Taipei City, Taiwan; 'Big Data Research Center, College of \\ Medicine, Fu-Jen Catholic University, New Taipei City, Taiwan; 'Division of Nephrology, Taipei Tzu Chi Hospital, \\ Buddhist Tzu Chi Medical Foundation, and School of Medicine, Buddhist Tzu Chi University, Hualien, Taiwan; \\ kDepartment of Microbiology \& Immunology, National Defense Medical Center, Taipei, Taiwan
}

\section{Keywords}

Carbon monoxide · Poisoning · Chronic kidney disease · Hyperbaric oxygen therapy

\begin{abstract}
Introduction: Patients with carbon monoxide poisoning (COP) commonly have long-term morbidities. However, it is not known whether patients with COP exhibit an increased risk of developing chronic kidney disease (CKD) and whether hyperbaric oxygen therapy (HBOT) alters this risk. Methods: This study identified 8,618 patients who survived COP and 34,464 propensity score-matched non-COP patients from 2000 to 2013 in a nationwide administrative registry. The primary outcome was the development of CKD. The association between COP and the risk of developing CKD was estimated using a Cox proportional hazards regression model; the cu-
\end{abstract}

mulated incidence of CKD among patients stratified by HBOT was evaluated using a Kaplan-Meier analysis. Results: After adjusting for covariates, the risk of CKD was 6.15-fold higher in COP patients than in non-COP controls. Based on the subgroup analyses, regardless of demographic characteristics, environmental factors, and comorbidities, the COP cohort exhibited an increased risk of developing CKD compared with the controls. The cumulative incidence of CKD in COP patients did not differ between the HBOT and nonHBOT groups ( $p=0.188$ ). Conclusions: COP might be an independent risk factor for developing CKD. Thus, clinicians should enhance the postdischarge follow-up of kidney function among COP patients.

(c) 2021 S. Karger AG, Basel

Wu-Chien Chien and Chia-Chao Wu contributed equally to this work.
Correspondence to:

Chia-ChaoWu,wucc@mail.ndmctsgh.edu.tw 


\section{Introduction}

Carbon monoxide poisoning (COP) remains a major cause of poisoning worldwide. In the USA, there are approximately 50,000 emergency department visits and 1,555 deaths per year due to COP $[1,2]$. Societal expense for accidental, nonfire, residential COP is approximately $\$ 3.47$ billion annually [3]. A similar burden affects the European region: a total of 140,490 carbon monoxide (CO)-related deaths (annual death rate of 2.24 per 100,000 population) were reported between 1980 and 2008 [4]. Thus, COP poses a significant economic and health burden.

Poisoning results from the inhalation of a high concentration of $\mathrm{CO}$, which has an absolute higher binding affinity to hemoglobin than oxygen. This causes tissue hypoxemia by forming carboxyhemoglobin, leading to a leftward shift of the oxyhemoglobin dissociation curve. Free CO causes intracellular and vascular oxidative stress, apoptosis, inflammation, and necrosis. These mechanisms contribute to remote organ injuries [5]. The recovery from COP is often complicated by the development of long-term morbidities or mortality. For example, myocardial injury [6] and neuropsychological sequelae [7-9] occurred in up to 37 and $40 \%$ of COP survivors, respectively. A large population study revealed an increased long-term mortality among COP survivors of moderate to severe degree [10]. Regarding kidney outcomes, few studies have identified an association between COP and acute kidney injury (AKI) [11-14]. Although most of these patients fully recovered from AKI, the development of AKI was strongly associated with subsequent mortality or neurological sequelae. However, there are still no literature data addressing whether COP causes the longterm kidney outcomes, particularly the development of chronic kidney disease (CKD).

CKD is another global public health issue that leads to serious sequelae such as adverse cardiovascular outcomes and death $[15,16]$. Notably, despite traditional risk factors, such as diabetes mellitus and hypertension, an increasing body of evidence indicates that $\mathrm{CO}$ could be a novel environmental risk factor for the development and progression of kidney disease [17]. An increased risk of an eGFR of $<60 \mathrm{~mL} / \mathrm{min} / 1.73 \mathrm{~m}^{2}$ and an eGFR decline of $>30 \%$ were associated with higher CO concentrations [18]. A COP event has an even higher concentration of $\mathrm{CO}$ exposure than an air pollutant. Whether COP serves as a novel CKD risk factor remains a subject of interest.

COP management comprises supplemental oxygen and hyperbaric oxygen therapy (HBOT) [8]. HBOT in- creases arterial and tissue oxygen tensions, accelerates CO removal, and elevates adenosine triphosphate production; it also reduces inflammation and oxidative stress [5]. The role of HBOT in COP management remains controversial [19], although some randomized trials suggest a potential benefit of reducing the long-term neurological sequelae $[8,20]$. Knowledge on the potential protective effects of HBOT on the kidneys has been restricted to animal models with AKI [21, 22]. Whether HBOT has longterm beneficial effects on kidney outcomes in humans remains unknown. This study aimed to assess the longterm risk of CKD and the efficacy of HBOT in patients with COP using Taiwan's National Health Insurance Research Database (NHIRD).

\section{Materials and Methods}

\section{Data Source}

Taiwan's National Health Insurance (NHI) program, a singlepayer mandatory health insurance program, was established in 1995 and enrolls $>99 \%$ of 23 million Taiwanese residents. The NHIRD, which is derived from registration files and original claim data of NHI beneficiaries, is structured for research purposes. It can illustrate the disease burden and health care process of virtually the entire population of Taiwan. The International Classification of Diseases, Ninth Edition, Clinical Modification (ICD-9$\mathrm{CM}$ ) was used for recording diagnoses in the NHIRD. This study used inpatient and outpatient datasets from patients enrolled in the Longitudinal Health Insurance Database of NHIRD during the period of 2000-2013. This cohort includes 989,753 individuals, who were randomly sampled beneficiaries from the original NHIRD in the year 2000. This study was approved by the Institutional Review Board of Tri-Service General Hospital (TSGH IRB No. 2-105-05-082).

\section{Study Design and Participants}

This was a retrospective matched-cohort study conducted between January 1, 2000, and December 31, 2013. The study cohort included 986,713 patients aged 18 years or older. Patients diagnosed with COP (ICD-9-CM 986) during this period were enrolled in the study group. The patient selection procedure is depicted in Figure 1. The index date was defined as the first clinical visit for COP. The comparison cohort (non-COP patients) was propensity score-matched with the COP patients at a ratio of 1:4 according to age, sex, and index date using the same exclusion criteria. We choose the propensity score matching by age and index date based on a prior epidemiological study conducted in Taiwan that reported an almost equal female and male distribution among 25,912 patients with COP and most of the patients were in the age groups of $20-35$ and $35-50$ years [23]. We included the index date because the incidence of unintentional and intentional COP changes over time. For example, in Taiwan, the annual unintentional mortality rate from COP significantly increased since 1997 [24], and the emergence of charcoal-burning suicide markedly contributed to the intentional COP cases since 2000 [25]. In addition, matching the index date results in equal follow-up peri- 


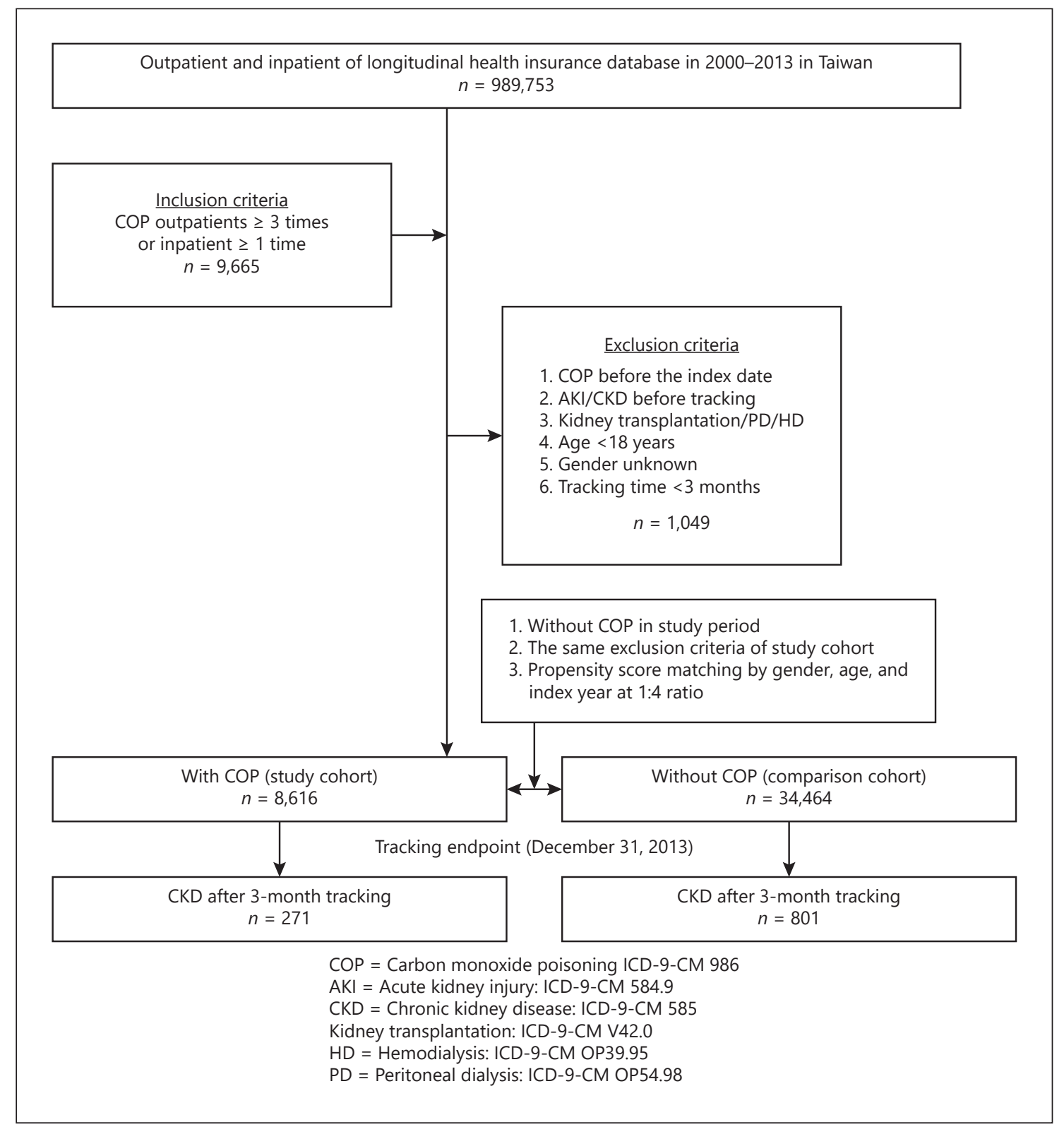

Fig. 1. Flowchart of study patient selection from the NHIRD in Taiwan. NHIRD, National Health Insurance Research Database.

ods for the primary outcome between the study and control groups. The exclusion criteria were as follows: COP diagnosis before the index date, AKI (ICD-9-CM 584.9) or CKD (ICD-9-CM 585) diagnosis before tracking, status of post-kidney transplantation (ICD-9-CM V42.0) or peritoneal dialysis (ICD-9-CM OP39.95) or hemodialysis (ICD-9-CM OP39.95), younger than 18 years, unknown gender, and tracking time $<3$ months. Based on these criteria, 8,616 patients with COP and 34,464 patients without COP were identified.

\section{Study Outcome}

The primary outcome was the development of CKD (ICD-9CM 585) since the index date. Patients in both the study and comparison cohorts were longitudinally traced from the index date until the end of 2013 or until one of the following events occurred: primary outcome or elimination because of lack of follow-up, death, or withdrawal from insurance. To confirm that patients had de novo CKD, we excluded patients who had been diagnosed with CKD or who underwent kidney replacement therapy (peritoneal dialysis, hemodialysis, or kidney transplantation) before tracking. A tracking time of $<3$ months after the index date was excluded to 
Table 1. Comparison of the demographics, characteristics, and comorbidities of the COP and non-COP cohorts

\begin{tabular}{|c|c|c|c|c|}
\hline \multirow[t]{2}{*}{ Variable } & \multirow[t]{2}{*}{ Total } & \multicolumn{2}{|l|}{ COP } & \multirow[t]{2}{*}{$p$ value } \\
\hline & & with & without & \\
\hline$N(\%)$ & 43,080 & $8,616(20.00)$ & $34,464(80.00)$ & \\
\hline Age, years & $38.73 \pm 13.97$ & $38.65 \pm 13.76$ & $38.75 \pm 14.02$ & 0.999 \\
\hline $18-29$ & $13,285(30.84)$ & $2,657(30.84)$ & $10,628(30.84)$ & \\
\hline $30-39$ & $12,555(29.14)$ & $2,511(29.14)$ & $10,044(29.14)$ & \\
\hline $40-49$ & $9,420(21.87)$ & $1,884(21.87)$ & $7,536(21.87)$ & \\
\hline $50-59$ & $4,460(10.35)$ & $892(10.35)$ & $3,568(10.35)$ & \\
\hline$\geq 60$ & $3,360(7.8)$ & $672(7.8)$ & $2,688(7.8)$ & \\
\hline Gender & & & & 0.999 \\
\hline Female & $20,745(48.15)$ & $4,149(48.15)$ & $16,596(48.15)$ & \\
\hline Male & $22,335(51.85)$ & $4,467(51.85)$ & $17,868(51.85)$ & \\
\hline \multicolumn{5}{|l|}{ Comorbidity } \\
\hline Hypertension & $2,195(5.10)$ & $434(5.04)$ & $1,761(5.11)$ & 0.804 \\
\hline DM & $2,035(4.72)$ & $387(4.49)$ & $1,648(4.78)$ & 0.268 \\
\hline $\mathrm{CHF}$ & $232(0.54)$ & $30(0.35)$ & $202(0.59)$ & $0.008^{*}$ \\
\hline Stroke & $921(2.14)$ & $131(1.52)$ & $790(2.29)$ & $<0.001^{*}$ \\
\hline COPD & $1,070(2.48)$ & $145(1.68)$ & $925(2.68)$ & $<0.001^{*}$ \\
\hline Liver cirrhosis & $1,725(4.00)$ & $138(1.60)$ & $1,587(4.60)$ & $<0.001^{*}$ \\
\hline CAD & $1,235(2.87)$ & $181(2.10)$ & $1,054(3.06)$ & $<0.001^{*}$ \\
\hline Arrhythmia & $574(1.33)$ & $90(1.04)$ & $484(1.40)$ & $0.009^{*}$ \\
\hline Hyperlipidemia & $835(1.94)$ & $70(0.81)$ & $765(2.22)$ & $<0.001^{*}$ \\
\hline CCI_R & $0.19 \pm 1.07$ & $0.08 \pm 0.70$ & $0.22 \pm 1.14$ & $<0.001^{*}$ \\
\hline Season & & & & $<0.001^{*}$ \\
\hline Spring (March-May) & $11,163(25.91)$ & $2,197(25.29)$ & $8,984(26.07)$ & \\
\hline Summer (June-August) & $10.565(24.52)$ & $1,681(19.51)$ & $8,884(25.78)$ & \\
\hline Autumn (September-November) & $10,081(23.40)$ & $1,940(22.52)$ & $8,141(23.62)$ & \\
\hline Winter (December-February) & $11,271(26.16)$ & $2,816(32.68)$ & $8,455(24.53)$ & \\
\hline Location & & & & $<0.001^{*}$ \\
\hline Northern Taiwan & $17,864(41.47)$ & $3,571(41.45)$ & $14,293(41.47)$ & \\
\hline Middle Taiwan & $12,176(28.26)$ & $2,827(32.81)$ & $9,349(27.13)$ & \\
\hline Southern Taiwan & $10,550(24.49)$ & $1,758(20.40)$ & $8,782(25.51)$ & \\
\hline Eastern Taiwan & $2,294(5.32)$ & $434(5.04)$ & $1,860(5.40)$ & \\
\hline Outlets Islands & $196(0.45)$ & $26(0.30)$ & $170(0.49)$ & \\
\hline Urbanization level & & & & $<0.001^{*}$ \\
\hline 1 (the highest) & $14,932(34.66)$ & $2,463(28.59)$ & $12,469(36.18)$ & \\
\hline 2 & $18,502(42.95)$ & $4,657(54.05)$ & $13,845(40.17)$ & \\
\hline 3 & $3,675(8.53)$ & $641(7.44)$ & $3,034(8.80)$ & \\
\hline 4 (the lowest) & $5,971(13.86)$ & $855(9.92)$ & $5,116(14.84)$ & \\
\hline Level of care & & & & $<0.001^{*}$ \\
\hline Medical center & $12,506(29.03)$ & $3,181(36.92)$ & $9,325(27.06)$ & \\
\hline Region hospital & $13.505(31.35)$ & $3,795(44.05)$ & $9,710(28.17)$ & \\
\hline Local hospital & $17,069(39.62)$ & $1,640(19.03)$ & $15,429(44.77)$ & \\
\hline
\end{tabular}

$\chi^{2}$ /Fisher's exact test; continuous variable: $t$ test. COP, carbon monoxide poisoning; CKD, chronic kidney disease; DM, diabetes mellitus; $\mathrm{CHF}$, congestive heart failure; COPD, chronic obstructive pulmonary disease; CAD, coronary artery disease; CCI_R, Charlson comorbidity index removed. ${ }^{*} p$ value $<0.05$.

ascertain that CKD developed after the COP event. In addition, to increase diagnostic accuracy, a CKD diagnosis should be confirmed at least 3 consecutive times at intervals of at least 3 months.

In the multivariate analysis model, we examined the impact of preexisting (prior to the index date) comorbidities, demographic characteristics, and environmental factors to investigate whether
COP is an independent risk factor for CKD. These comorbidities included diabetes mellitus (ICD-9-CM 250), hypertension (ICD9-CM 401-405), heart failure (ICD-9-CM 428), coronary artery disease (ICD-9-CM 410-414), arrhythmia (ICD-9-CM 427), hyperlipidemia (ICD-9-CM 272), stroke (ICD-9-CM 430-438), liver cirrhosis (ICD-9-CM 571), and chronic obstructive pulmonary 
Fig. 2. Kaplan-Meier analysis of the cumulative incidence of $\mathrm{CKD}$ between the patients with COP (solid line) and without COP (dashed line) cohorts (log-rank $p<$ 0.001). CKD, chronic kidney disease; COP, carbon monoxide poisoning.

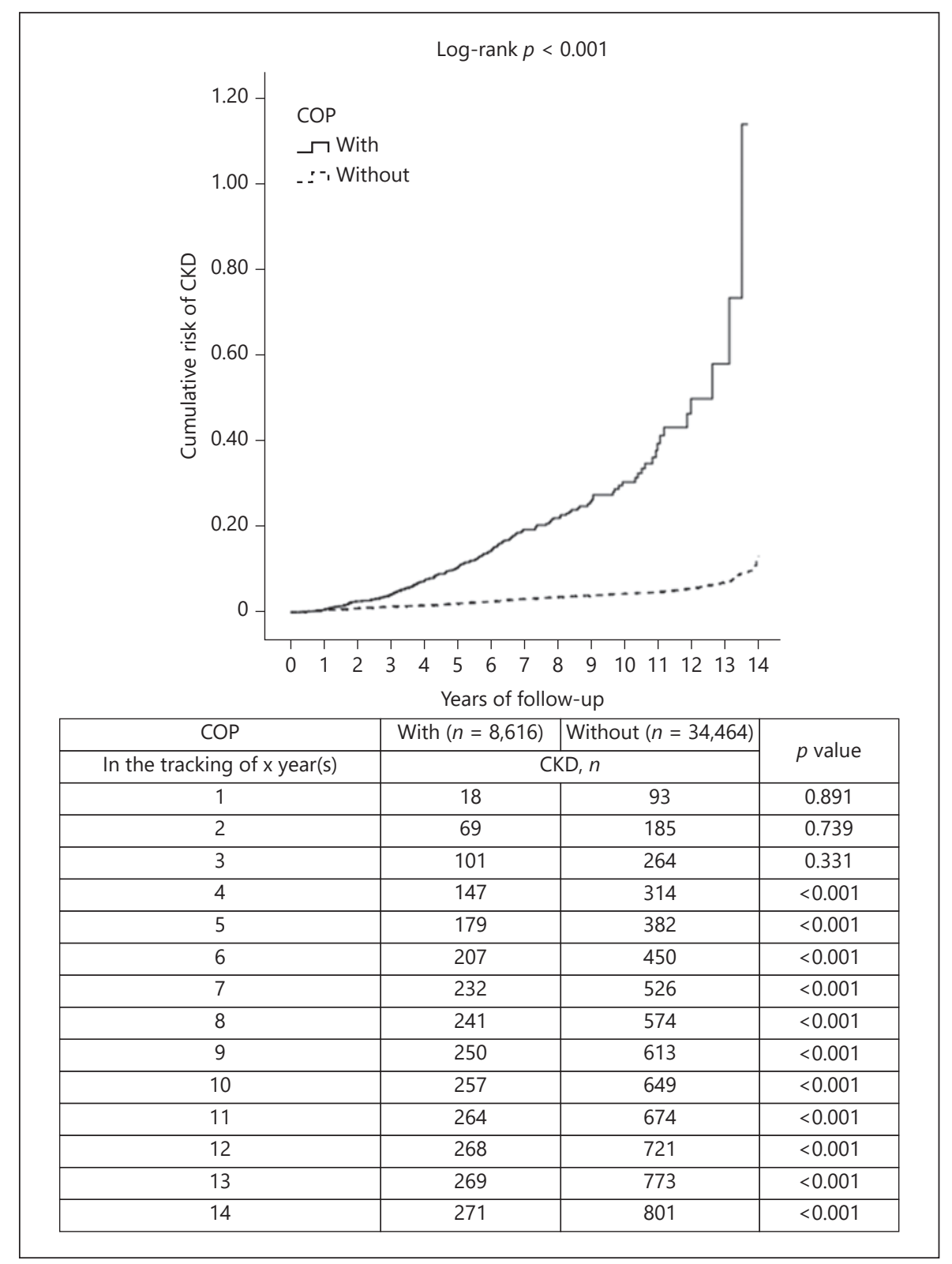

disease (ICD-9-CM 490-496). Demographic characteristics and environmental factors included age, sex, urbanization level, season, and hospital level of care.

The COP patients were further divided into subgroups according to HBOT. To evaluate the therapeutic effects of HBOT on the kidneys, we compared the cumulative incidence of CKD in both the study (with HBOT group) and comparison groups (without HBOT).

\section{Statistical Analysis}

The baseline distributions of demographic characteristics and the comorbidities of the COP patients and non-COP patients were described using percentages and means \pm standard deviation for the categorical and continuous variables, respectively. Differences between the study and comparison cohorts were compared using the $\chi^{2}$ test for categorical variables and the $t$ test for continuous variables, as appropriate.

The multivariable Cox proportional hazards model was used to estimate the adjusted hazard ratios (HRs) of the primary outcome. We further performed a subgroup analysis to determine the adjusted HRs of the primary outcome in the COP and non-COP patients. A Kaplan-Meier analysis with the log-rank test was used to compare the cumulative incidences of CKD over time between the COP and non-COP patients and between subgroups of COP patients stratified by HBOT. All data were analyzed using SAS statistical software (version 9.3 for Windows). A 2-tailed $p<0.05$ was considered significant. 
Table 2. Comparison of the incidence and adjusted $\mathrm{HR}$ for $\mathrm{CKD}$ in the COP and non-COP cohorts stratified by variables listed in the table by using Cox regression

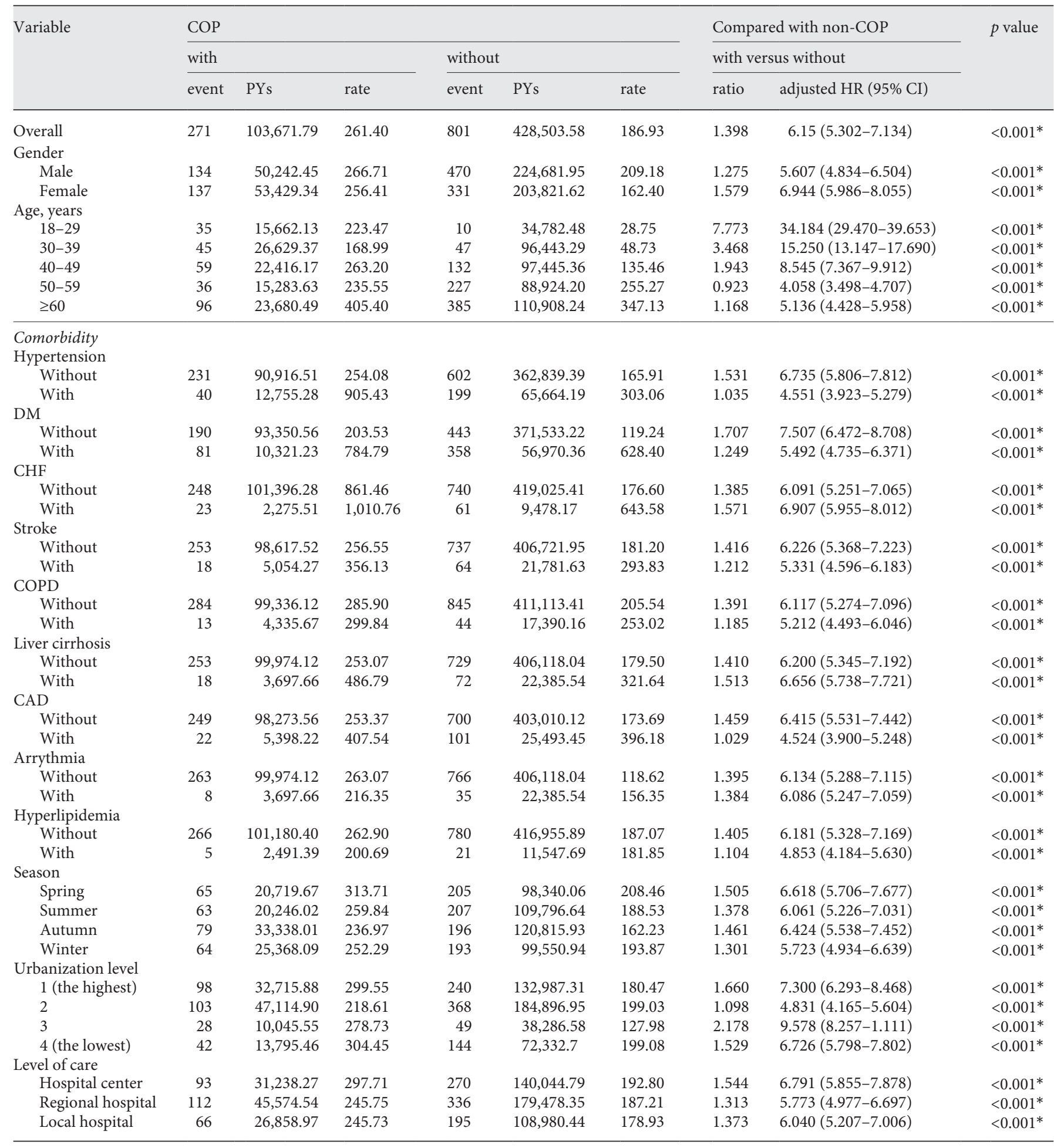

Rate, incidence rate (per $10^{5} \mathrm{PYs}$ ); ratio, rate in cases $\div$ rate in controls; adjusted HR, adjusted for all the variables listed in the table: gender, age, comorbidities (hypertension, diabetes, CHF, stroke, COPD, liver cirrhosis, CAD, arrythmia, and hyperlipidemia), season, urbanization level and level of care. PYs, person-years; HR, hazard ratio; CKD, chronic kidney disease; COP, carbon monoxide poisoning; DM, diabetes mellitus; CHF, congestive heart failure; COPD, chronic obstructive pulmonary disease; CAD, coronary artery disease. ${ }^{*} p$ value $<0.05$. 


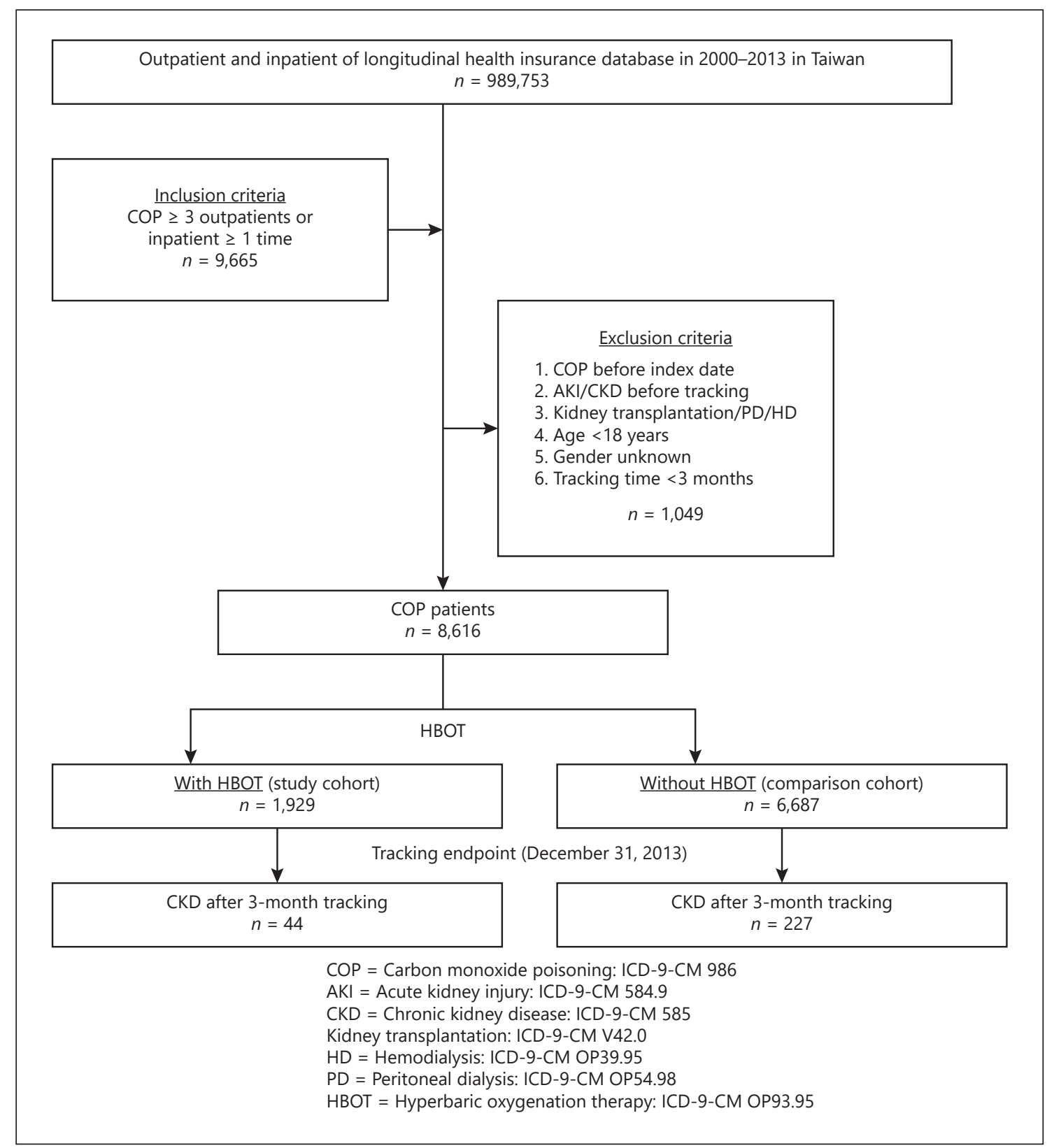

Fig. 3. Flowchart of COP patients stratified by HBOT. COP, carbon monoxide poisoning; HBOT, hyperbaric oxygen therapy.

\section{Results}

A total of 8,616 COP patients who met the inclusion and exclusion criteria were enrolled in this study between January 1, 2000, and December 31, 2013. After applying propensity score matching, a total of 34,464 matched controls were enrolled. The baseline characteristics and comorbidities of the study cohorts categorized by COP are presented in Table 1 . In both cohorts, the mean patient age was 38 years and $51.8 \%$ of the patients were male. The incidences of hypertension and diabetes mellitus, the major risk factors of CKD, did not differ significantly between the 2 cohorts. The following comorbidities occurred less frequently in the COP patients than in the non-COP cohort: congestive heart failure ( 0.35 vs. $0.59 \%$; $p=0.008)$, stroke (1.52 vs. $2.29 \%$; $p<0.001)$, COPD (1.68 


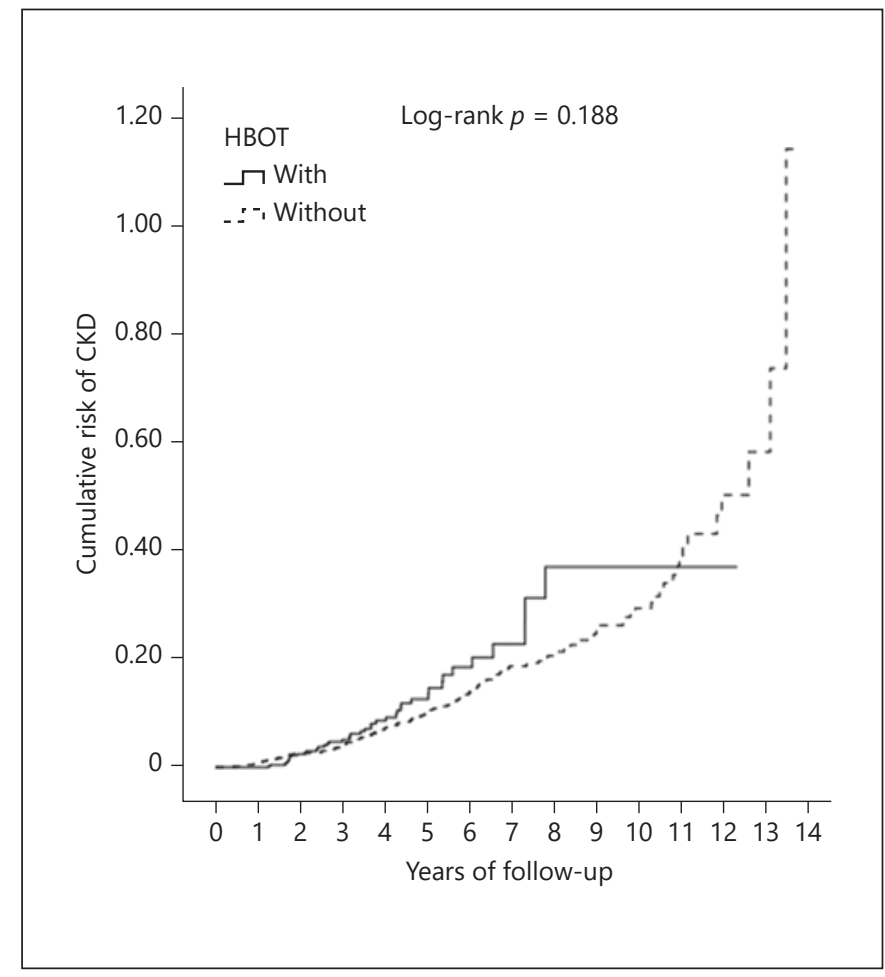

Fig. 4. Kaplan-Meier analysis of the cumulative incidence of CKD between patients with HBOT and without HBOT among the COP cohort. CKD, chronic kidney disease; COP, carbon monoxide poisoning; HBOT, hyperbaric oxygen therapy.

vs. $2.68 \%$; $p<0.001)$, liver cirrhosis ( 1.60 vs. $4.60 \%$; $p<$ $0.001)$, coronary artery disease ( 2.10 vs. $3.06 \% ; p<0.001)$, arrhythmia ( 1.04 vs. $1.40 \% ; p=0.009$ ), and hyperlipidemia ( 0.81 vs. $2.22 \%$; $p<0.001)$. COP tended to be diagnosed more frequently during the winter (32.68 vs. $24.53 \% ; p<0.008)$ and among people living in an area with an urbanization level of 2 (54.05 vs. $40.17 \%$; $p<$ $0.001)$.

During the 14-year follow-up, 271 (3.14\%) of the 8,616 COP patients and 801 (2.32\%) of 34,464 non-COP patients developed CKD (Fig. 1). The mean follow-up time and time between COP and de novo CKD among COP and control groups are presented in online suppl. Table 1 (see www.karger.com/doi/10.1159/000515383 for all online suppl. material). The median mortality age (approximately 60 years) did not differ between the groups (online suppl. Table 2). The Kaplan-Meier curve for the cumulative incidence of CKD stratified by COP with a log-rank test is shown in Figure 2. COP patients had a significantly increased incidence of CKD events (log-rank, $p<0.001$ ) when measured over 4 years after the COP index date.

Carbon Monoxide Poisoning and Chronic Kidney Disease
The adjusted HR of the CKD events in the groups of subjects with COP and the matched controls are presented in Table 2. After adjusting for age, sex, preexisting comorbidities, the Charlson comorbidity index score, season, urbanization level, and level of care, the risk of CKD remained higher in the COP cohort (adjusted HR: 6.15, 95\% CI 5.302-7.134). This finding was confirmed with sensitivity and subgroup analyses. After stratification, the increased risk of CKD was consistent across sex, age, comorbidities (Table 2), season, urbanization level, and level of care. This indicates that COP might be an independent risk factor of developing CKD.

To further explore the risk of CKD with the use of HBOT in COP patients, we stratified COP patients by HBOT use as depicted in Figure 3. Of the COP patients, 1,929 received $\mathrm{HBOT}$, whereas 6,687 did not. During the 14-year follow-up, we identified 44 (2.28\%) CKD events in the HBOT group and 227 (3.39\%) CKD events in the non-HBOT group. The cumulative incidence of CKD did not differ significantly between the HBOT group and the non-HBOT group (log-rank, $p=0.188$ ) (Fig. 4). Thus, HBOT might not alter the risk of developing CKD in the COP cohort.

\section{Discussion}

This national population-based observational matched-cohort study demonstrated that the COP cohort was associated with a 6.15-fold increase in the risk of developing CKD compared with the non-COP cohort after adjusting for numerous covariates. This finding was confirmed with sensitivity and subgroup analyses. We noted that, even in patients without comorbidities or with different demographic characteristics and environmental factors, the risks of CKD remained higher after COP. This indicates that COP might be an independent risk factor for CKD. Furthermore, our study noted the lack of an association between HBOT and the risk of CKD among COP cohorts. This might indicate that the toxicological effects of COP on the kidneys precede the therapeutic effects of HBOT, and these effects might be irreversible or partially reversible.

Few cohort studies have identified an association between COP and kidney outcomes. Kim et al. [13] reported that AKI occurred in approximately $18 \%$ of patients with nonfire-related COP and was mainly detected on the first day of admission. Although most of these patients fully recovered from AKI, the development and progression of AKI have prognostic values for neurological defi- 
cits or mortality [13]. Two previous studies revealed higher rates of AKI when including all etiologies of COP patients or when enrolling only patients with intentional COP. $\mathrm{Ku}$ et al. [11] reported that approximately $23 \%$ of COP survivors developed AKI after CO exposure. The other study by Chen et al. [12] reported that up to $39 \%$ of patients experienced AKI complications after charcoalburning suicide, and AKI was again a strong predictor of mortality. A recent study by Pan et al. [14] reported that approximately $18 \%$ of patients with COP developed poor outcomes, which was defined as having sequelae, being bedridden, or death. In this group, blood urea nitrogen and serum creatinine levels were significantly higher on arrival [14]. Although these studies did not address COinduced long-term kidney outcomes, the development of AKI was linked to subsequent mortality. One study also demonstrated that patients who recovered from AKI are at an increased risk of the long-term incidence of stroke, and its impact was similar to that of diabetes [26]. These effects indicate that AKI might trigger a cascade of nonresolvable perturbations that may involve remote organs. The likely pathogenic mechanisms may be related to factors such as oxidative stress, inflammation, uremic toxins, endothelial dysfunction, or cell cycle disruption [2729]. Each of these factors may also lead to an ongoing state that predisposes the patient to the development of further kidney injury. Indeed, AKI, even with recovery, is gradually being recognized as a contributing factor to the development of CKD $[27,30]$. For example, several clinical observational studies in adults and children have reported an increased long-term incidence of CKD after functional recovery from an episode of AKI [31-34]. The present study found an increased incidence of CKD in the COP cohort. Although this might be partially explained by AKI, ongoing effort is necessary to understand the causality, because a single episode of AKI manifests differently in time, and the outcomes are related to AKI severity, clinical treatment, and host risk factors [35]. It is difficult to differentiate the proportion of COP patients who had AKI and then directly progressed to CKD from the patients who had AKI, with kidney function recovery, and then progressed to CKD based solely on ICD diagnostic codes. Furthermore, many factors such as residual confounding, ascertainment bias, and misclassification of the exposure and outcome, should be considered [36]. Therefore, further prospective, longitudinal, and control studies that rigorously assess kidney function in all participants may elucidate the causal nature of these findings.
Another question regarding COP and the risk for CKD is whether COP causes kidney damage or whether this simply represents an associative relationship. This study demonstrates the role of COP in the burden of CKD. The associated higher risk of CKD attributable to COP was persistent after accounting for diabetes mellitus, hypertension, and comorbidities that are considered to be drivers of kidney disease, which suggests that the burden of CKD is likely due to factors other than these traditional risk factors (Table 2). Of interest, an increasing body of evidence has shown the association between major air pollutants including ambient concentrations of $\mathrm{CO}$ and kidney disease [18, 37, 38]. Exposure to excess concentrations of $\mathrm{CO}$ is associated with an increased risk of developing incident CKD, an eGFR decline of $>30 \%$, and endstage renal disease [18]. Although the exact mechanism of how CO is involved in the pathogenesis is not clear, there are several possibilities. One potential biological mechanism is that $\mathrm{CO}$, similar to inhaled pollutants, might trigger systemic inflammation and systemic oxidative stress via the lungs, which in turn induces endothelial injury, atherosclerotic disease, and distant organ damage including the kidneys [5, 39-42]. For example, air pollution exposure was related to increased levels of endothelial microparticles, antiangiogenic and inflammatory mediators (such as TNF $\alpha$ and IL-6), hematologic factors, plasma viscosity, and suppressed proangiogenic growth factors. These responses contribute to the pathogenic sequelae of atherogenesis and may increase the thrombotic potential [41-43]. The release of such proinflammatory cytokines, oxidative stress, and endothelial dysfunction were strongly associated with a higher level of albuminuria and a lower eGFR and the risk of the development and progression of CKD [44-47]. COP might be an initial trigger of this process. The second proposed mechanism may be associated with tissue hypoxia and cell damage. $\mathrm{CO}$ traverses the alveolar space and enters the circulation where CO binds to hemoglobin irreversibly with an absolute higher binding affinity than that of $\mathrm{CO}$ to oxygen, causing hypoxemia. Free CO can bind to heme proteins such as cytochrome c oxidase, which impairs mitochondrial function and thereby contributes to energy depletion and hypoxia $[5,48]$. The limited kidney tissue oxygen supply makes the kidney susceptible to hypoxia and has long been recognized as a crucial factor in the pathogenesis of AKI, which is a risk factor for CKD, as discussed earlier [49]. The pathophysiology of COP on kidney damage may be multifactorial. It is plausible that one or more of these mechanistic pathways may explain the association described here. 
Although treating COP patients with HBOT remains controversial, HBOT is still commonly used as a primary or adjunctive therapy for COP patients $[8,19]$. In this study, the cumulative incidence of developing CKD among the COP patients did not differ significantly regardless of whether HBOT was administered. This result may be attributable to the following possibilities. First, although HBOT preconditioning has been shown to offer protective effects on AKI in animal models of ischemiareperfusion kidney injury $[21,22]$, there is limited evidence regarding the effect of $\mathrm{HBOT}$ on $\mathrm{AKI}$ in humans. In the COP cohort, AKI often developed before HBOT administration. In accordance with the earlier discussion, AKI, even treated or in recovery, still carries a high risk of developing CKD. Although HBOT reduced the toxicological effects of $\mathrm{CO}$, these effects could have permanently damaged cells, leading to chronic consequences. However, a limitation of this explanation is that we cannot clarify whether the proportions of COP patients who had AKI in the HBOT and non-HBOT cohorts are comparable or whether HBOT is correlated with the severity of disease among the COP cohort. Because of selection bias, there is a possibility that the sickest patients are never able to receive HBOT, which would confound the association of HBOT with outcomes. Second, the pathophysiology of COP on kidney damage may be multifactorial and HBOT may reverse some but not all the mechanistic pathways.

This study has some limitations. First, detailed information regarding the patients' current use of vasoactive or potential nephrotoxic medications, which might have influenced the primary outcomes, were difficult to entirely obtain from the NHIRD database. Second, certain detailed risk factors for CKD, such as dietary habits, body mass index, or smoking, are lacking, which could result in residual confounding bias. For example, dietary habits such as high salt and low potassium diets are important risk factors for hypertension, cardiovascular disease, and CKD [50]. Consequently, to minimize the potential confounding effect, we adjusted for these factors by including related diseases, such as hypertension, cardiovascular disease, and smoking-related comorbidities. Third, detailed information that may illustrate the chronic complications of COP including blood and urine laboratory data were unavailable in the NHIRD database. Fourth, early-stage CKD may be underestimated because certain early-stage CKD patients who only have mild anatomical or physiologic abnormalities (such as proteinuria) with normal serum creatinine levels may be misclassified in terms of their outcomes. In addition, there may be a detection bias. Patients who survived COP are more likely to visit hospi- tals for various sequelae, which may increase the probability of detecting CKD. Fifth, mortality can become a competing risk factor for the development of CKD. For example, higher mortality or earlier death may alter the incidence of CKD. In this study, the mean patient age in the COP cohort was 38 years and the median mortality age was 60 years (online suppl. Table 2). This indicates that most patients complete adequate follow-up. Therefore, we assumed that bias due to mortality was low. Sixth, certain variables in the patient characteristics were not balanced, which may have contributed to bias. Although the initial balance was not perfect, the incidences of hypertension and diabetes mellitus, the major risk factors of CKD, did not differ significantly between the 2 cohorts. Of note, fewer chronic comorbidities were observed in $\mathrm{COP}$ patients. Conversely, the risk of developing CKD was higher in the COP group than in the non-COP group. This indirectly suggests that the burden of CKD is likely due to factors other than the traditional CKD risk factors and partially supports that COP might be an independent risk factor for CKD. Seventh, the retrospective design typically had unmeasured confounding factors compared with prospective studies. Therefore, further large-scale prospective studies are necessary to elucidate the causalities. In addition, detailed group differences in the HBOT and non-HBOT cohorts should be investigated in future studies. Considering that COP may be associated with coexposure to other pollutants that induce organ injuries, future studies could incorporate data from the Environmental Protection Agency monitoring stations across Taiwan to determine the levels of exposure to air pollutants and establish a causal relationship more precisely. The main strength of this study is that it utilizes a nationwide population-based design with a large number of patients enrolled and performs a longitudinal evaluation of the long-term impact of COP and HBOT on the development of CKD.

In summary, our research demonstrates a possible association between COP and the risk of developing CKD particularly when measured over 4 years after a COP event. The increased CKD risk was consistent across all subgroups of interest. However, a significant association was not observed between HBOT and the incidence of CKD among COP patients. Although the exact mechanisms that underlie this association require ongoing study, our study findings suggest that all patients with COP, regardless of the presence or extent of traditional CKD risk factors, should be closely monitored for subsequent kidney function. 


\section{Acknowledgements}

We appreciate the Health and Welfare Data Science Center, Ministry of Health and Welfare, Taiwan, for providing the $\mathrm{Na}$ tional Health Insurance Research Database (NHIRD).

\section{Statement of Ethics}

This study was approved by the Institutional Review Board of Tri-Service General Hospital (TSGH IRB No. 2-105-05-082). All information in the analyzed database indicating patient identity was removed before analysis; hence, the review board waived the requirement for written informed consent of the patients.

\section{Conflict of Interest Statement}

All authors declare that they have no conflict of interest.

\section{Funding Sources}

This study was supported by the Tri-Service General Hospital Research Foundation (TSGH-C05-110033 and TSGH-B-110012), and the sponsor has no role in the study design, data collection and analysis, decision to publish, or preparation of the manuscript

\section{Author Contributions}

Study design: Kuang-Yu Wei, Chen-Yi Liao, and Chia-Chao Wu. Data curation: Chi-Hsiang Chung and Wu-Chien Chien. Formal analysis: Kuang-Yu Wei, Chi-Hsiang Chung, and Wu-Chien Chien. Methodology: Fu-Huang Lin, Chang-Huei Tsao, Chien-An Sun, and Wu-Chien Chien. Project administration: Kuang-yu Wei and Chen-Yi Liao. Resources: Wu-Chien Chien. Software: ChiHsiang Chung. Supervision: Kuo-Cheng Lu, Wu-Chien Chien, and Chia-Chao Wu. Writing original draft: Kuang-Yu Wei. Review and editing: Kuo-Cheng $\mathrm{Lu}, \mathrm{Wu}$-Chien Chien, and ChiaChao Wu. All authors approved the manuscript.

\section{References}

1 Hampson NB. U.S. mortality due to carbon monoxide poisoning, 1999-2014. accidental and intentional deaths. Ann Am Thorac Soc. 2016 Oct;13(10):1768-74.

2 Hampson NB, Weaver LK. Carbon monoxide poisoning: a new incidence for an old disease. Undersea Hyperb Med. 2007 May-Jun;34(3): $163-8$.

3 Hampson NB. Cost effectiveness of residential carbon monoxide alarms. Undersea Hyperb Med. 2017 Sept-Oct;44(5):393-7.

4 Braubach M, Algoet A, Beaton M, Lauriou S, Héroux ME, Krzyzanowski M. Mortality associated with exposure to carbon monoxide in WHO European Member States. Indoor Air. 2013 Apr;23(2):115-25.

5 Weaver LK. Clinical practice. Carbon monoxide poisoning. N Engl J Med. 2009 Mar 19; 360(12):1217-25

6 Henry CR, Satran D, Lindgren B, Adkinson C, Nicholson CI, Henry TD. Myocardial injury and long-term mortality following moderate to severe carbon monoxide poisoning. JAMA. 2006 Jan 25;295(4):398-402.

7 Ernst A, Zibrak JD. Carbon monoxide poisoning. N Engl J Med. 1998 Nov 26;339(22): 1603-8.

8 Weaver LK, Hopkins RO, Chan KJ, Churchill S, Elliott CG, Clemmer TP, et al. Hyperbaric oxygen for acute carbon monoxide poisoning. N Engl J Med. 2002 Oct 3;347(14):1057-67.

9 Weaver LK, Valentine KJ, Hopkins RO. Carbon monoxide poisoning: risk factors for cognitive sequelae and the role of hyperbaric oxygen. Am J Respir Crit Care Med. 2007 Sep 1; 176(5):491-7.

10 Hampson NB, Rudd RA, Hauff NM. Increased long-term mortality among survivors of acute carbon monoxide poisoning. Crit Care Med. 2009 Jun;37(6):1941-7.
$11 \mathrm{Ku} \mathrm{CH}$, Hung HM, Leong WC, Chen HH, Lin JL, Huang WH, et al. Outcome of patients with carbon monoxide poisoning at a far-east poison center. PLoS One. 2015;10(3): e0118995.

12 Chen YC, Tseng YC, Huang WH, Hsu CW, Weng $\mathrm{CH}$, Liu SH, et al. Acute kidney injury predicts mortality after charcoal burning suicide. Sci Rep. 2016 Jul 19;6:29656.

13 Kim YJ, Sohn CH, Seo DW, Oh BJ, Lim KS, Chang JW, et al. Analysis of the development and progression of carbon monoxide poisoning-related acute kidney injury according to the Kidney Disease Improving Global Outcomes (KDIGO) criteria. Clin Toxicol. 2018 Aug;56(8):759-64.

14 Pan KT, Shen CH, Lin FG, Chou YC, Croxford B, Leonardi G, et al. Prognostic factors of carbon monoxide poisoning in Taiwan: a retrospective observational study. BMJ Open. 2019 Nov 18;9(11):e031135.

15 Go AS, Chertow GM, Fan D, McCulloch CE, Hsu CY. Chronic kidney disease and the risks of death, cardiovascular events, and hospitalization. N Engl J Med. 2004 Sep 23;351(13): 1296-305.

16 Collaboration GBDCKD. Global, regional, and national burden of chronic kidney disease, 1990-2017: a systematic analysis for the Global Burden of Disease Study 2017. Lancet. 2020 Feb 29;395(10225):709-33.

17 Afsar B, Elsurer Afsar R, Kanbay A, Covic A, Ortiz A, Kanbay M. Air pollution and kidney disease: review of current evidence. Clin Kidney J. 2019 Feb;12(1):19-32.
18 Bowe B, Xie Y, Li T, Yan Y, Xian H, Al-Aly Z. Associations of ambient coarse particulate matter, nitrogen dioxide, and carbon monoxide with the risk of kidney disease: a cohort study. Lancet Planet Health. 2017 Oct;1(7): e267-76.

19 Buckley NA, Juurlink DN, Isbister G, Bennett $\mathrm{MH}$, Lavonas EJ. Hyperbaric oxygen for carbon monoxide poisoning. Cochrane Database Syst Rev. 2011 Apr 13(4):CD002041.

20 Thom SR, Taber RL, Mendiguren II, Clark JM, Hardy KR, Fisher AB. Delayed neuropsychologic sequelae after carbon monoxide poisoning: prevention by treatment with hyperbaric oxygen. Ann Emerg Med. 1995 Apr; 25(4):474-80.

21 Migita H, Yoshitake S, Tange Y, Choijookhuu $\mathrm{N}$, Hishikawa Y. Hyperbaric oxygen therapy suppresses apoptosis and promotes renal tubular regeneration after renal ischemia/reperfusion injury in rats. Nephrourol Mon. 2016 Jan;8(1):e34421.

22 Kovacevic S, Ivanov M, Miloradovic Z, Brkic P, Vajic UJ, Zivotic M, et al. Hyperbaric oxygen preconditioning and the role of NADPH oxidase inhibition in postischemic acute kidney injury induced in spontaneously hypertensive rats. PLoS One. 2020;15(1):e0226974.

23 Huang CC, Ho CH, Chen YC, Lin HJ, Hsu CC, Wang JJ, et al. Demographic and clinical characteristics of carbon monoxide poisoning: nationwide data between 1999 and 2012 in Taiwan. Scand J Trauma Resusc Emerg Med. 2017 Jul 14;25(1):70.

24 Shie HG, Li CY. Population-based case-control study of risk factors for unintentional mortality from carbon monoxide poisoning in Taiwan. Inhal Toxicol. 2007 Aug;19(10): 905-12. 
25 Chang SS, Chen YY, Yip PS, Lee WJ, Hagihara A, Gunnell D. Regional changes in charcoal-burning suicide rates in East/Southeast Asia from 1995 to 2011: a time trend analysis. PLoS Med. 2014 Apr;11(4):e1001622.

$26 \mathrm{Wu} \mathrm{VC}, \mathrm{Wu} \mathrm{PC}, \mathrm{Wu} \mathrm{CH}$, Huang TM, Chang $\mathrm{CH}$, Tsai PR, et al. The impact of acute kidney injury on the long-term risk of stroke. J Am Heart Assoc. 2014 Jul 15;3(4):e000933.

27 Chawla LS, Kimmel PL. Acute kidney injury and chronic kidney disease: an integrated clinical syndrome. Kidney Int. 2012 Sep; 82(5):516-24.

28 Wu VC, Young GH, Huang PH, Lo SC, Wang $\mathrm{KC}$, Sun CY, et al. In acute kidney injury, indoxyl sulfate impairs human endothelial progenitor cells: modulation by statin. Angiogenesis. 2013 Jul;16(3):609-24.

29 Lisowska-Myjak B. Uremic toxins and their effects on multiple organ systems. Nephron Clin Pract. 2014;128(3-4):303-11.

30 Coca SG, Singanamala S, Parikh CR. Chronic kidney disease after acute kidney injury: a systematic review and meta-analysis. Kidney Int. 2012 Mar;81(5):442-8.

31 Wald R, Quinn RR, Luo J, Li P, Scales DC, Mamdani MM, et al. Chronic dialysis and death among survivors of acute kidney injury requiring dialysis. JAMA. 2009 Sep 16; 302(11):1179-85.

32 Bucaloiu ID, Kirchner HL, Norfolk ER, Hartle JE 2nd, Perkins RM. Increased risk of death and de novo chronic kidney disease following reversible acute kidney injury. Kidney Int. 2012 Mar;81(5):477-85.

33 Jones J, Holmen J, De Graauw J, Jovanovich A, Thornton S, Chonchol M. Association of complete recovery from acute kidney injury with incident CKD stage 3 and all-cause mortality. Am J Kidney Dis. 2012 Sep;60(3):4028 .
34 Mammen C, Al Abbas A, Skippen P, Nadel H, Levine D, Collet JP, et al. Long-term risk of CKD in children surviving episodes of acute kidney injury in the intensive care unit: a prospective cohort study. Am J Kidney Dis. 2012 Apr;59(4):523-30.

35 Thakar CV, Christianson A, Himmelfarb J, Leonard AC. Acute kidney injury episodes and chronic kidney disease risk in diabetes mellitus. Clin J Am Soc Nephrol. 2011 Nov; 6(11):2567-72.

36 Rifkin DE, Coca SG, Kalantar-Zadeh K. Does AKI truly lead to CKD? J Am Soc Nephrol. 2012 Jun;23(6):979-84.

37 Bowe B, Xie Y, Li T, Yan Y, Xian H, Al-Aly Z. Particulate matter air pollution and the risk of incident CKD and progression to ESRD. J Am Soc Nephrol. 2018 Jan;29(1):218-30.

38 Kim HJ, Min JY, Seo YS, Min KB. Association between exposure to ambient air pollution and renal function in Korean adults. Ann Occup Environ Med. 2018;30:14.

39 Sorensen M, Daneshvar B, Hansen M, Dragsted LO, Hertel O, Knudsen L, et al. Personal PM2.5 exposure and markers of oxidative stress in blood. Environ Health Perspect. 2003 Feb;111(2):161-6.

40 Sun Q, Wang A, Jin X, Natanzon A, Duquaine $\mathrm{D}$, Brook RD, et al. Long-term air pollution exposure and acceleration of atherosclerosis and vascular inflammation in an animal model. JAMA. 2005 Dec 21;294(23):3003-10.

41 Ruckerl R, Hampel R, Breitner S, Cyrys J, Kraus U, Carter J, et al. Associations between ambient air pollution and blood markers of inflammation and coagulation/fibrinolysis in susceptible populations. Environ Int. 2014 Sep;70:32-49.
42 Pope CA 3rd, Bhatnagar A, McCracken JP, Abplanalp W, Conklin DJ, O'Toole T. Exposure to fine particulate air pollution is associated with endothelial injury and systemic inflammation. Circ Res. 2016 Nov 11;119(11): 1204-14.

43 Peters A, Döring A, Wichmann HE, Koenig W. Increased plasma viscosity during an air pollution episode: a link to mortality? Lancet. 1997 May 31;349(9065):1582-7.

44 Kao MP, Ang DS, Pall A, Struthers AD. Oxidative stress in renal dysfunction: mechanisms, clinical sequelae and therapeutic options. J Hum Hypertens. 2010 Jan;24(1):1-8.

45 Malyszko J. Mechanism of endothelial dysfunction in chronic kidney disease. Clin Chim Acta. 2010 Oct 9;411(19-20):1412-20.

46 Shankar A, Sun L, Klein BE, Lee KE, Muntner $\mathrm{P}$, Nieto FJ, et al. Markers of inflammation predict the long-term risk of developing chronic kidney disease: a population-based cohort study. Kidney Int. 2011 Dec;80(11): 1231-8.

47 Gupta J, Mitra N, Kanetsky PA, Devaney J, Wing MR, Reilly M, et al. Association between albuminuria, kidney function, and inflammatory biomarker profile in CKD in CRIC. Clin J Am Soc Nephrol. 2012 Dec; 7(12):1938-46.

48 Alonso JR, Cardellach F, López S, Casademont J, Miró O. Carbon monoxide specifically inhibits cytochrome c oxidase of human mitochondrial respiratory chain. Pharmacol Toxicol. 2003 Sep;93(3):142-6.

49 Eckardt KU, Bernhardt WM, Weidemann A, Warnecke C, Rosenberger C, Wiesener MS, et al. Role of hypoxia in the pathogenesis of renal disease. Kidney Int Suppl. 2005 Dec(99):S4651.

50 Wei KY, Gritter M, Vogt L, de Borst MH, Rotmans JI, Hoorn EJ. Dietary potassium and the kidney: lifesaving physiology. Clin Kidney J. 2020 Dec;13(6):952-68. 